Check for updates

Cite this: RSC Adv., 2019, 9, 3012

\title{
Dual-functional melanin-based nanoliposomes for combined chemotherapy and photothermal therapy of pancreatic cancer
}

\author{
Jian Wang, $\uparrow^{\text {abcd }}$ Jiasui Chai, $\dagger^{\mathrm{e}}$ Lei Liu,,$^{\text {bcdf }}$ Zilin Cui, $^{\text {abcd }}$ Dongming Duan, ${ }^{\text {abcd }}$ \\ Rui Shi ${ }^{\text {abcd }}$ and Yamin Zhang (D)*abcd
}

Pancreatic cancer, one of the most common gastrointestinal tract cancers, leads to a high mortality rate of over $80 \%$ among patients. Conventional chemotherapy with gemcitabine (GEM) is undesirable due to the lack of effective tumor accumulation. To improve the survival of pancreatic cancer patients and the therapeutic efficiency of chemotherapy, dual-functional melanin-based nanoliposomes loaded with GEM were synthesized in our study, which combined chemotherapy and photothermal therapy (PTT). Hypothermia caused by melanin under near-infrared (NIR) laser exerted detrimental damage on pancreatic cancer cells after the passive accumulation of nanoliposomes in the tumor sites. Besides, the temperature increase could enhance the release of GEM from the nanoliposomes by changing the structural integrity of the nanoliposomes. Therefore, a synergistic antitumor effect was achieved by loading the chemotherapy agent GEM and the photothermal agent melanin into the nanoliposomes. The findings in this study strongly support that melanin-based nanoliposomes could be a desirable strategy against pancreatic carcinoma.

Received 15th November 2018 Accepted 26th December 2018

DOI: $10.1039 / \mathrm{c} 8 \mathrm{ra09420a}$

rsc.li/rsc-advances on the enhanced permeability and retention (EPR) effect, leading to a high concentration of antitumor agents remaining in the tumor sites. ${ }^{13}$ Nanoliposomes have great advantages of controllable drug encapsulation, good biocompatibility and prolonged circulation cycle. ${ }^{\mathbf{1 4 1 5}}$ To pursue higher therapeutic efficiency against tumors and higher biosafety, responsivereleased nanoliposome systems have been synthesized in the last few years. Various types of smart-responsive systems have been studied with triggers for controllable release in the tumor including $\mathrm{pH},{ }^{16}$ light,${ }^{17}$ enzymes,${ }^{18}$ ultrasonic, ${ }^{19}$ magnetism, ${ }^{20}$ and heat. ${ }^{21}$ Among them, thermosensitive nanoliposomes were prepared on the basis of the hyperthermia-triggered mechanism. It should be mentioned that the typical characteristics of the microenvironment are mostly hypovascular. ${ }^{22,23}$ Hyperthermia caused by a photothermal agent can be a desirable method to increase tumor blood flow and microvascular permeability, ${ }^{\mathbf{2 4 2 5}}$ which may facilitate the penetration of chemotherapeutic agents like GEM into the tumor tissue. Besides, hyperthermia produced under near-infrared (NIR) light can trigger apoptosis and coagulative necrosis in tumor cells. $^{26,27}$

Melanin mainly exists in many parts of human beings, including in the skin, hair, and eyes. ${ }^{28}$ Recent studies have shown that melanin-based drug carrier systems can serve as a promising form of photothermal therapy because of the optical absorption capability and native biocompatibility. ${ }^{29-31}$ To improve its therapeutic efficiency against pancreatic cancer, a novel melanin-based nanoliposome was constructed via a thin-film evaporation method in this study. The 
nanoliposome achieved combined chemo- and photothermal therapy by entrapping the chemotherapeutic agent GEM in the hydrophobic phase and photothermal agent melanin in the hydrophilic phase. Under NIR irradiation, hyperthermia and the controlled release of GEM from the nanoliposome exerted a synergistic antitumor effect on pancreatic cells. With great biocompatibility, the melanin-based nanoliposome had been proven as a desirable nanoplatform for treating pancreatic cancer.

In our study, novel nanoliposomes co-encapsulated with GEM, which was a therapeutic agent for advanced pancreatic cancer, and melanin, which exhibited a photothermal effect under NIR light, were synthesized. The as-prepared GEM-MelLip was capable of accumulating into the tumor site selectively owing to the enhanced EPR effect. After entering the tumor cells, melanin in the nanoliposomes converted absorbed light energy into heat upon NIR laser radiation, which led to apoptosis and the coagulative necrosis of the tumor cells. In addition, the phospholipid fluidity of GEM-Mel-Lip increased under hyperthermia generated by the melanin, resulting in the release of GEM and the following cancer cells killing effect. GEM-Mel-Lip provided dual-functional treatment combining chemotherapy and photothermal therapy for pancreatic carcinoma (Scheme 1).

\section{Results and discussion}

\section{Design, synthesis, and characterization of GEM-Mel-Lip}

The characterization of the tested nanoliposomes was shown in Table 1. The diameters of tested nanoliposomes were about $220 \mathrm{~nm}$, which indicated that both melanin and GEM loading exerted no effect on the size of nanoliposomes. It should be mentioned that larger particles were more feasible to permeate into the vessels in tumors than that in normal tissues. The asprepared nanoliposomes with sizes of about $220 \mathrm{~nm}$ in this study tend to accumulate in the tumor sites. Both GEM-Lip and GEM-Mel-Lip achieved high encapsulation efficiency (EE > $80 \%)$. The size change of GEM-Mel-Lip over a two-week period was shown in Fig. 1. It was found that the size remained almost unchanged during the two-week period, which illustrated the high stability of GEM-Mel-Lip.

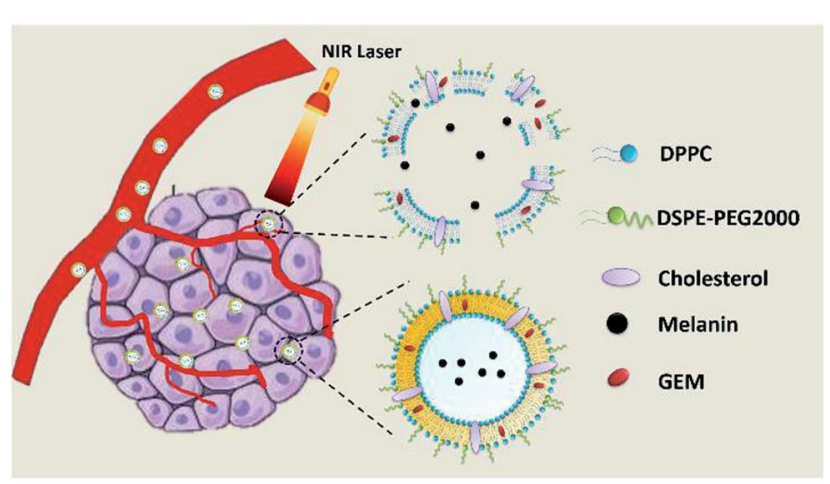

Scheme 1 Schematic of the antitumor effect of the as-synthesized GEM-Mel-Lip.
Table 1 Characterization results of the blank Lip, GEM-Lip, Mel-Lip, and GEM-Mel-Lip

\begin{tabular}{llll}
\hline Nanoliposomes & Diameter $(\mathrm{mm})$ & PDI & EE (\%) \\
\hline Blank Lip & $215.2 \pm 2.2$ & $0.22 \pm 0.08$ & - \\
GEM-Lip & $218.5 \pm 2.9$ & $0.16 \pm 0.06$ & $81.7 \pm 4.8$ \\
Mel-Lip & $221.2 \pm 3.1$ & $0.12 \pm 0.09$ & - \\
GEM-Mel-Lip & $220.2 \pm 3.4$ & $0.17 \pm 0.01$ & $82.2 \pm 5.2$
\end{tabular}

The absorption spectra of GEM-Mel-Lip, GEM, and melanin were presented in Fig. 2. As shown in Fig. 2, melanin exhibited broad and strong absorption in the NIR region from $300 \mathrm{~nm}$ to $900 \mathrm{~nm}$. A broad absorption curve was monitored for GEM-MelLip, which was attributed to the loading of melanin onto the nanoliposomes. GEM showed an absorbance spectrum peak at $268 \mathrm{~nm}$. Compared to melanin, GEM-Mel-Lip induced a new absorbance peak at $268 \mathrm{~nm}$, which corresponded well to that of GEM, suggesting the successful loading of GEM onto the nanoliposomes. The above results supported the successful encapsulation of GEM and melanin onto the nanoliposomes.

The zeta potentials of melanin, blank Lip, and GEM-Mel-Lip were investigated in this study. According to Fig. 3, melanin was negatively charged. It was found that blank Lip showed a negatively charged surface, which may be because DSPE-PEG 2000 didn't fully cover the surface of the nanoliposomes. GEM-MelLip presented a more negative zeta potential, which may due to the slightly slow diffusion of melanin onto the surface of the

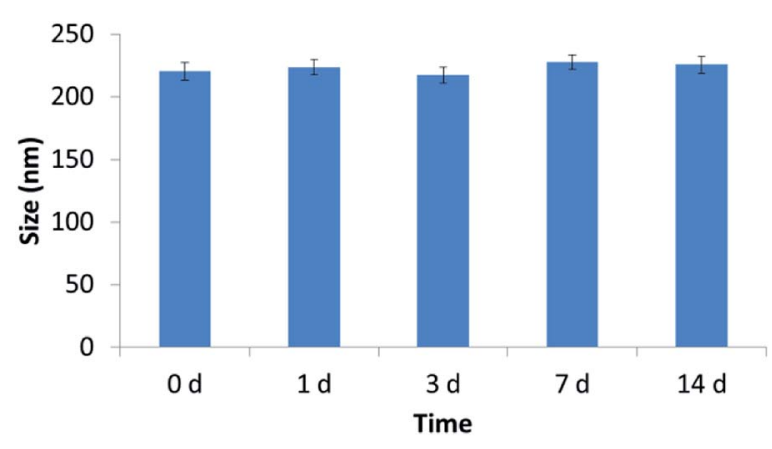

Fig. 1 Size change of GEM-Mel-Lip during a two-week period.

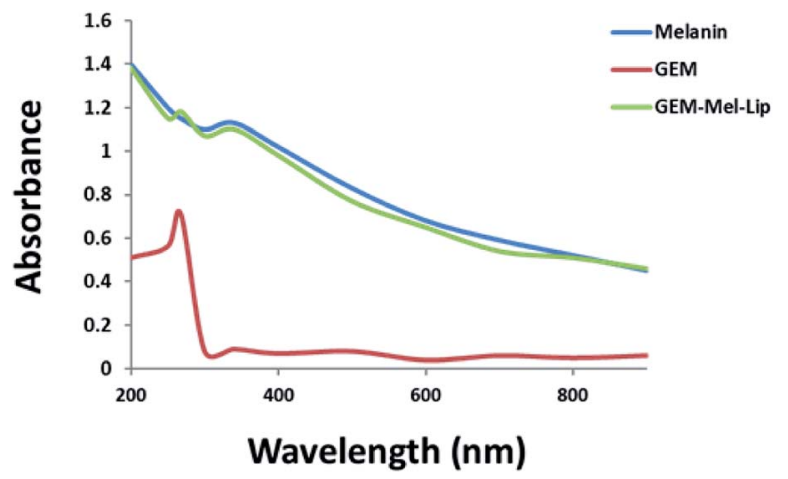

Fig. 2 The UV-vis absorption spectra of GEM-Mel-Lip, GEM, and melanin. 


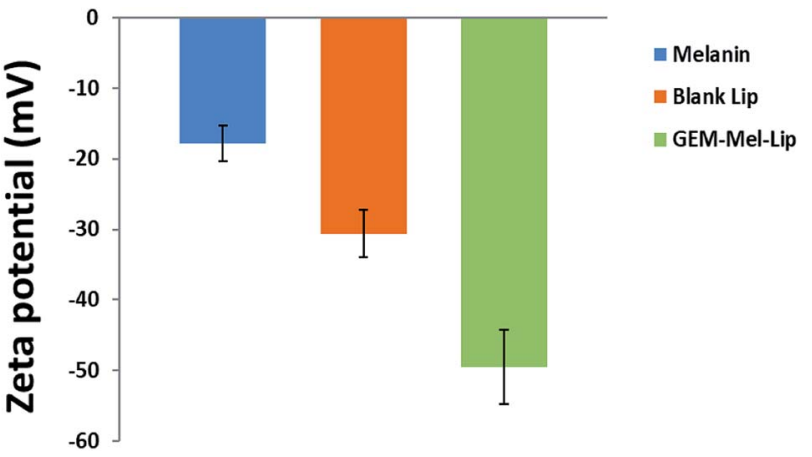

Fig. 3 Zeta potential of melanin, blank Lip, and GEM-Mel-Lip.

nanoliposomes. It also proved the successful loading of melanin.

The TEM images of blank Lip and GEM-Mel-Lip were shown in Fig. 4. Both represented uniform spherical morphology, indicating that loading with GEM and Mel in the nanoliposomes had no significant influence on the morphology.

\section{In vitro photothermal properties of the tested nanoliposomes}

The photothermal conversion effect of melanin in the NIR range endowed the GEM-Mel-Lip with higher therapeutic efficiency compared to GEM-Lip. As shown in Fig. 5, the temperature of GEM-Mel-Lip could increase up to $58.5{ }^{\circ} \mathrm{C}$ within $5 \mathrm{~min}$ of NIR irradiation at a power density of $1.50 \mathrm{~W} \mathrm{~cm}{ }^{-2}$. No significant temperature change was observed in the GEM-Lip group. This indicated that the presence of melanin could efficiently convert NIR light into thermal energy. The photothermal transduction
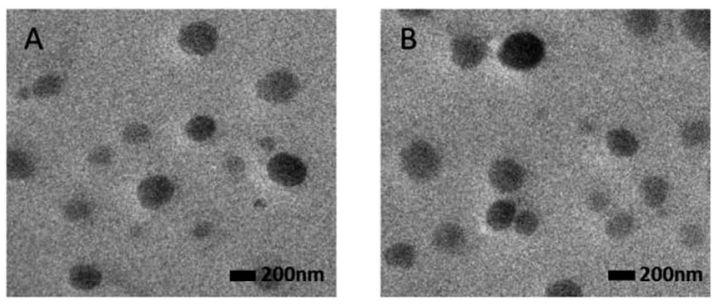

Fig. 4 TEM image of blank Lip (A) and GEM-Mel-Lip (B) (scale bar = $200 \mathrm{~nm})$.

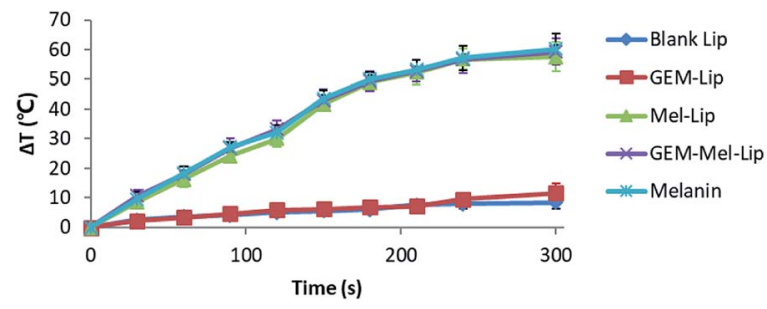

Fig. 5 Plot of temperature change of blank Lip, GEM-Lip (GEM concentration: $20 \mu \mathrm{g} \mathrm{mL}^{-1}$ ), Mel-Lip (melanin concentration: $180 \mu \mathrm{g}$ $\mathrm{mL}^{-1}$ ), and GEM-Mel-Lip (GEM concentration: $20 \mu \mathrm{g} \mathrm{mL}^{-1}$, melanin concentration: $180 \mu \mathrm{g} \mathrm{mL}^{-1}$ ) under NIR irradiation $(808 \mathrm{~nm}$ ) for $5 \mathrm{~min}$ at a power density of $1.50 \mathrm{~W} \mathrm{~cm}^{-2}$. of melanin showed no significant difference with Mel-Lip or GEM-Mel-Lip, which meant that encapsulation by nanoliposomes exerted no effect on the photothermal properties of melanin. We also compared the photothermal conversion capabilities of GEM-Mel-Lip with that of Mel-Lip and blank Lip. It was found that the temperature increase of GEM-Mel-Lip was much higher than that of blank Lip but showed no significant difference from Mel-Lip, suggesting the loading of GEM exerted no negative influence on the photothermal conversion efficiency of melanin.

\section{In vitro GEM release profiles}

GEM release profiles of GEM-Lip and GEM-Mel-Lip with or without NIR treatment were investigated in this study (Fig. 6). Slow drug release was observed for GEM-Lip and GEM-Mel-Lip when incubated for $24 \mathrm{~h}$ without NIR irradiation, suggesting the minor drug leakage from both nanoliposomes without the appropriate stimulating factor. Then, the nanoliposomes were exposed to NIR irradiation for $5 \mathrm{~min}$. After irradiation, the release profile of GEM-Mel-Lip was distinctively different from that of GEM-Lip. The released amounts of GEM in GEM-Mel-Lip reached nearly $80 \%$ within $24 \mathrm{~h}$. The rapid release of GEM in GEM-Mel-Lip under NIR irradiation was probably because the generated hyperthermia destroyed the structural integrity of nanoliposomes, which led to the rapid leakage of the loaded drug. Moreover, the majority of GEM in GEM-Lip still remained inside the matrix, which suggested that the slower release profile was due to the lack of a drug release mechanism. It had been reported that tumor cells should be exposed to a maximum concentration of the therapeutic agent when maximum therapeutic effects were expected. ${ }^{32}$ The rapid release of GEM-Mel-Lip under NIR irradiation would lead to a high concentration of GEM accumulating in the tumor sites, achieving a desirable antitumor effect.

\section{In vitro cytotoxicity and cellular uptake studies}

The cytotoxicity to the pancreatic cancer cells of GEM-Lip, MelLip, and GEM-Mel-Lip with or without NIR irradiation was evaluated by a typical CCK-8 assay in this study. As is shown in Fig. 7A, cell viability decreased as the concentration of nanoliposomes increased. However, without NIR irradiation, all

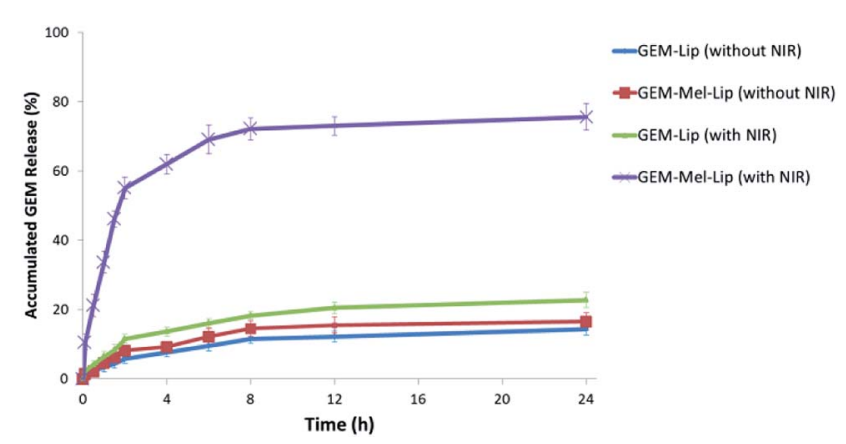

Fig. 6 Release profiles of GEM from GEM-Lip and GEM-Mel-Lip with or without NIR treatment $\left(808 \mathrm{~nm}, 5 \mathrm{~min}, 1.50 \mathrm{~W} \mathrm{~cm}^{-2}\right)$. 
A

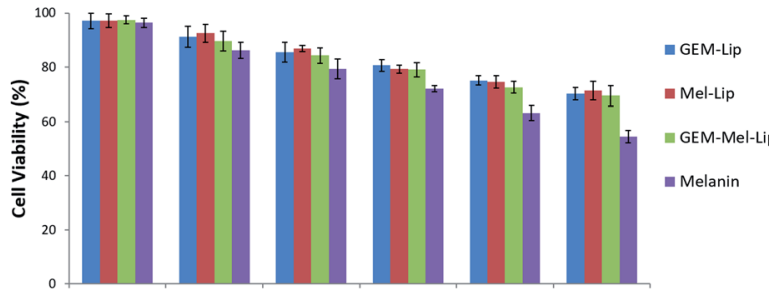

B

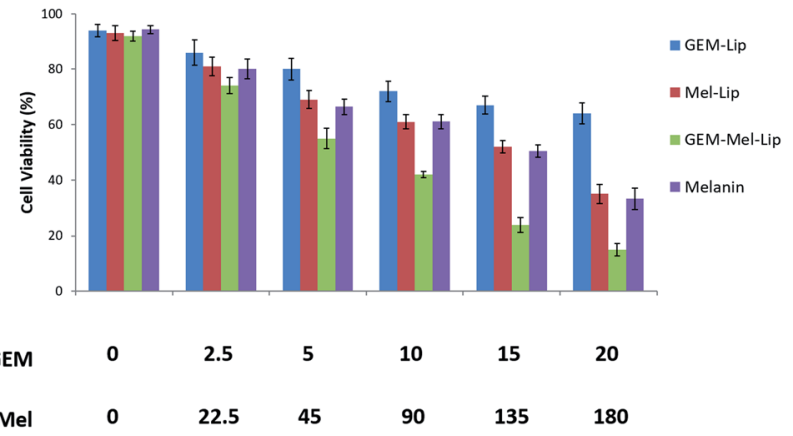

Concentration $(\mu \mathrm{g} / \mathrm{mL})$

Fig. 7 Cell cytotoxicity of GEM-Lip, Mel-Lip, GEM-Mel-Lip, and melanin after co-incubation with BxPC-3 cells for $24 \mathrm{~h}$ without (A) and with (B) NIR irradiation at different concentrations.

tested nanoliposomes indicated limited cytotoxicity to BxPC-3 cells with more than $70 \%$ cell viability even when nanoliposomes were administered at a concentration of $200 \mu \mathrm{g}$ $\mathrm{mL}^{-1}$. The high survival rate in the Mel-Lip group proved that no photothermal damage occurred in the BxPC-3 cells in the absence of NIR irradiation. It had been reported that free melanin exerted hypotoxicity towards cancer cells. ${ }^{33}$ We also found that free melanin showed minor toxic effect with elevated concentration. The encapsulation of melanin in the nanoliposomes could reduce hypotoxicity. The high cell viability of Mel-Lip in this study demonstrated that encapsulating melanin into the nanoliposomes could largely reduce the cytotoxicity of melanin, which suggested the minor leakage of melanin from the liposomes. Similar behavior was observed when it came to GEM-Lip and GEM-Mel-Lip. This suggested that the release of GEM from the matrix was limited under physiological conditions.

The cytotoxicity of the tested nanoliposomes under NIR irradiation was shown in Fig. 7B. The distinctive behavior of cytotoxicity was described when Mel-Lip and GEM-Mel-Lip were exposed to NIR irradiation, while the cell viability of the GEMLip group did not experience any significant change. Fewer cancer cells incubated with Mel-Lip (<40\%) and GEM-Mel-Lip $(<20 \%)$ survived when compared with the no NIR irradiation case. The low survival rate in the Mel-Lip group signified that the photothermal effect of melanin under NIR irradiation showed a detrimental effect on BxPC-3 cells. GEM-Mel-Lip showed higher cytotoxicity than Mel-Lip, indicating that the loaded GEM from the carrier could be controllably released by hyperthermia, which was generated by melanin. Therefore, the combination of GEM and melanin would significantly improve the antitumor effect on pancreatic cancer cells.
Cellular uptake studies with or without NIR irradiation were taken to investigate the targeting capability of GEM-Mel-Lip. Fig. 8 shows representative photomicrographs of fluorescence images of BxPC-3 cells incubated with GEM-Mel-Lip. Red fluorescence was observed in the cytoplasm of the BxPC-3 cells despite the presence of NIR irradiation, suggesting that GEMMel-Lip had good targeting capability toward pancreatic cancer cells. Compared to the no NIR group, a higher red fluorescence intensity was observed in the NIR group. The enhancement of fluorescence intensity may be because more DiO was released from GEM-Mel-Lip owing to the generated hyperthermia under NIR irradiation. Therefore, GEM-Mel-Lip could be efficiently endocytosed into BxPC-3 cells, ensuring high photothermal efficiency and the effective release of loaded GEM for killing BXPC-3 cells.

\section{In vivo therapeutic efficacy of GEM-Mel-Lip}

To assess the antitumor efficacy of GEM-Mel-Lip in vivo, BxPC-3 tumor-bearing mice were randomly separated into 7 groups: saline (control), GEM-Lip ( \pm NIR), Mel-Lip $( \pm$ NIR), and GEMMel-Lip $( \pm$ NIR). Mice were intravenously injected with the above drug formulations when the tumor volumes reached $\sim 100 \mathrm{~mm}^{3}$. The tumors were irradiated with an $808 \mathrm{~nm}$ laser at $1.5 \mathrm{~W} \mathrm{~cm}^{-2}$ for $5 \min (1 \mathrm{~min} \times 5$ times, 1 min intervals). The tumor volume changes of the 7 groups were monitored, and the weight of each mouse was recorded every three days.

As is shown in Fig. 9A, the tumor volume of the control group reached $2634 \pm 336 \mathrm{~mm}^{3}$ after treatment for 21 days. In the no NIR irradiation case, a certain tumor inhibition was achieved for the GEM-Lip (-NIR) group, Mel-Lip (-NIR) group, and GEM-Mel-Lip (-NIR) group. No significant differences between the GEM-Lip (-NIR) group and the GEM-Mel-Lip (-NIR) group were observed, indicating that the release of GEM in nanoliposomes was limited owing to the integrity of the structure. When exposed to NIR irradiation, the tumor volume in GEM-Lip (+NIR) group was much higher than that in the GEM-Mel-Lip (+NIR) group, which was about $234 \pm 132 \mathrm{~mm}^{3}$. The Mel-Lip (+NIR) group showed a higher tumor inhibition rate than the Mel-Lip (-NIR) group, which proved the high photothermal effect on pancreatic cancer cells. The GEM-Mel-Lip (+NIR) group was much smaller in size than the Mel-Lip (+NIR) group. The above results proved the advantage of combinational

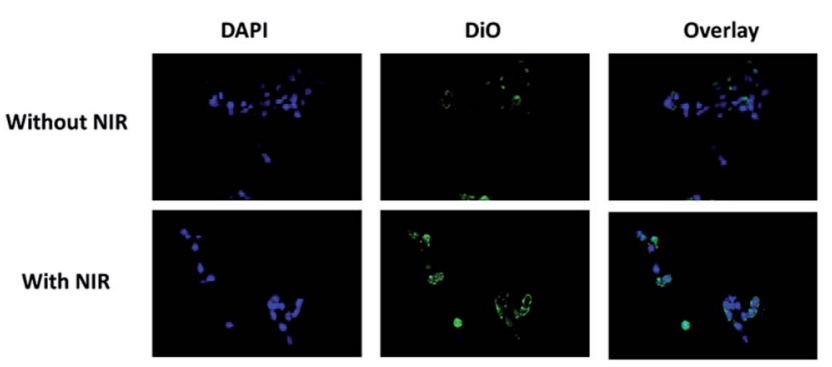

Fig. 8 Intracellular uptake profiles of BxPC-3 cells incubated with GEM-Mel-Lip with or without NIR irradiation $(808 \mathrm{~nm}, 5 \mathrm{~min}, 1.50 \mathrm{~W}$ $\mathrm{cm}^{-2}$ ), as observed by CLSM. 

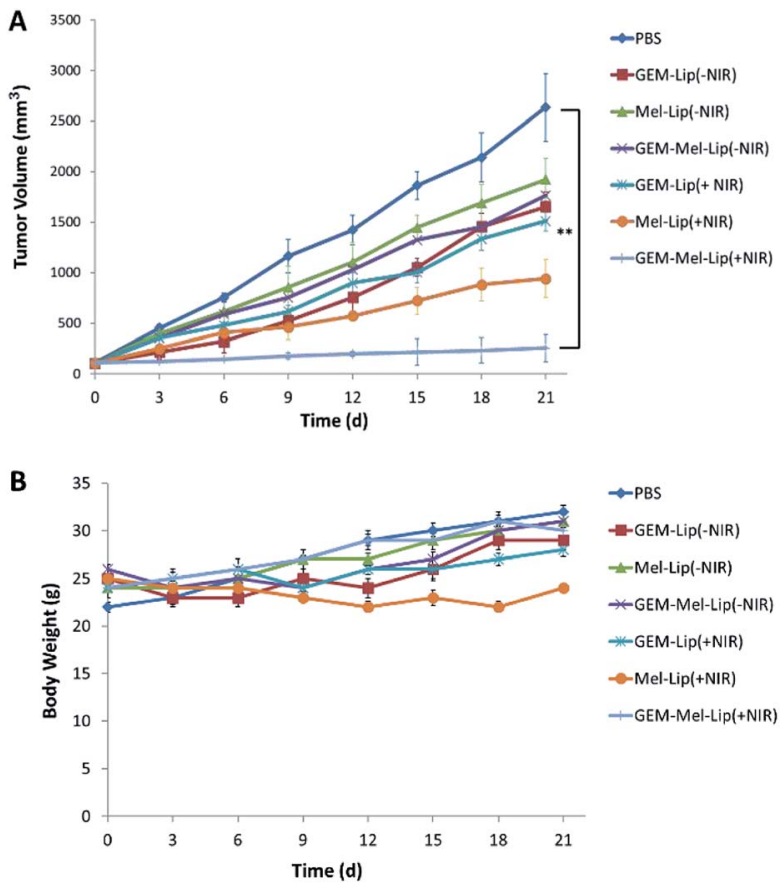

Fig. 9 In vivo antitumor effects of various treatments on BxPC-3 tumor-bearing mice. (A) Tumor growth curve following different treatments $(n=5)$ (B) body weight as a function of days post-treatment for the indicated treatment groups. ${ }^{* *} P<0.01$.

thermal therapy and chemotherapy over monotherapy, which relied on single GEM or photothermal therapy.

To further study the antitumor effect of tested nanoliposomes, H\&E staining tests of tumor sections were taken. As shown in Fig. 10, when compared between the GEM-Lip ( \pm NIR) group, Mel-Lip (-NIR) group, and GEM-Mel-Lip (-NIR) group, more deformed nuclei were observed in the H\&E-stained tumor section of the GEM-Mel-Lip (+NIR) group and the Mel-Lip (+NIR) group. This indicated that hyperthermia generated in the tumor sites after NIR irradiation could lead to high apoptosis and coagulative necrosis of the cancer cells.

\section{In vivo safety of GEM-Mel-Lip}

The safety of a promising treatment combined with photothermal therapy and chemotherapy should be evaluated in vivo.
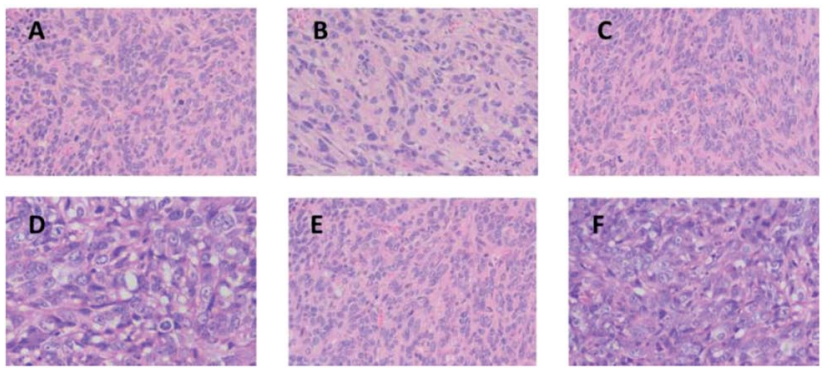

Fig. $10 \mathrm{H}$ EE staining on tumor sections after various treatments. (A) GEM-Lip (-NIR) group, (B) GEM-Lip (-NIR) group, (C) Mel-Lip (-NIR) group, (D) Mel-Lip (+NIR) group, (E) GEM-Mel-Lip (-NIR) group, (F) GEM-Mel-Lip (+NIR) group.

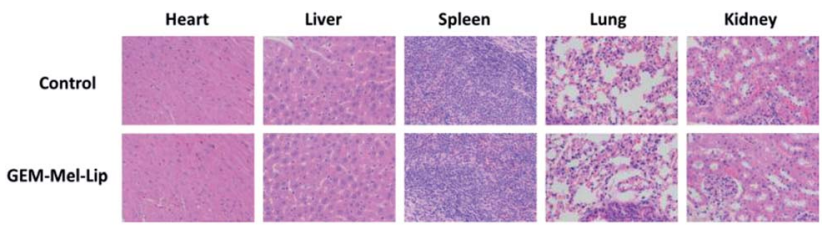

Fig. 11 H\&E staining of the major organs (heart, liver, spleen, lung, and kidney) of BxPC-3 tumor-bearing nude mice in the control group and the GEM-Mel-Lip group.

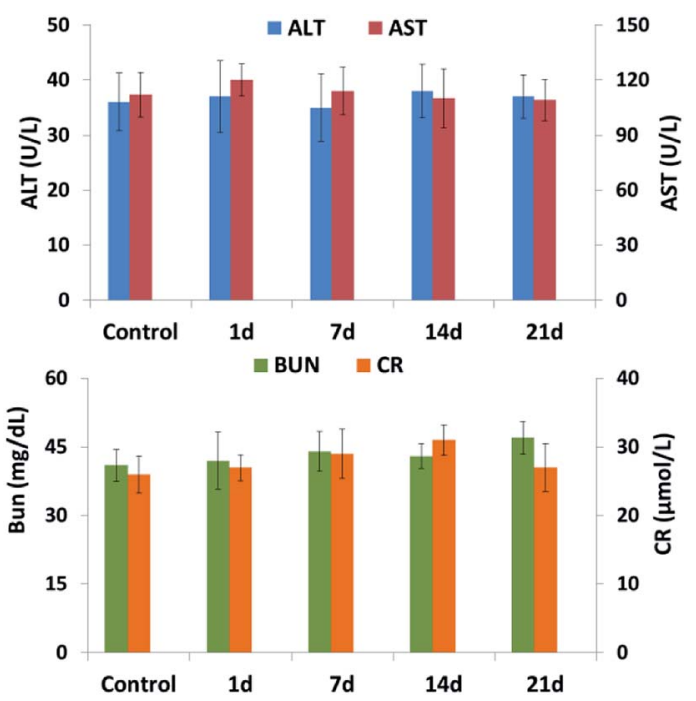

Fig. 12 Hematological assay of BxPC-3 tumor-bearing nude mice from the control group and the GEM-Mel-Lip group every 1 day, 3 days, 7 days, and 14 days post intravenous injection of GEM-Mel-Lip.

Fig. 9B showed that the weight of mice in the GEM-Mel-Lip groups did not bear significant loss, indicating that GEM-MelLip exerted no systemic toxicity to BxPC-3 tumor-bearing mice.

H\&E staining of the main organs from the GEM-Mel-Lip group was performed to further detect the toxicity of GEMMel-Lip. As shown in Fig. 11, no irregularities were observed in the heart, liver, spleen, lung, and kidney tissues. Considering nanocarriers with sizes of about $200 \mathrm{~nm}$ were cleared by liver and kidney, a high concentration of GEM-Mel-Lip loaded with chemotherapy agent and melanin would accumulate in the liver and kidney. Hepatic and renal function was also inspected by analyzing the liver functional marker (ALT, AST) and kidney functional markers (BUN, CR) after treatment with GEM-MelLip. It turned out that treatment with GEM-Mel-Lip showed no damage to the liver or kidney (Fig. 12), suggesting the efficient attenuation of GEM-Mel-Lip to the side effects of chemotherapy. In summary, the results above proved that GEM-MelLip possessed good biocompatibility.

\section{Conclusions}

In this study, we successfully designed dual-functional nanoliposomes (GEM-Mel-Lip) with high photothermal therapy and chemotherapy efficiency to treat pancreatic cancer, which was systematically demonstrated both in vitro and in vivo. Besides, 
GEM-Mel-Lip was proven to be highly safe for pancreatic treatment. GEM-Mel-Lip was prepared by encapsulating GEM in the hydrophobic phase and melanin in the hydrophilic phase of the nanoliposomes. Under NIR irradiation, following two aspects of therapeutic effect worked: (1) hyperthermia generated from melanin had an antitumor efficacy on the pancreatic tumor cells, (2) the release of GEM from the nanoliposomes were controlled and enhanced in the tumor sites via hyperthermia. It proved to be a desirable strategy against pancreatic carcinoma through the synergistic effect of the photothermal effect and combination therapy.

\section{Experimental}

\section{Materials}

Cholesterol (Chol) was purchased from New Probe (Beijing, China). 1,2-Dipalmitoyl-sn-glycero-3-phosphocholine (DPPC) and 1,2-distearoylsn-glycero-3-phosphoethanolamine- $N$ [methoxy(polyethyleneglycol)-2000] (DSPE-PEG2000) were purchased from Meilun Biotechnology Co., LTD (Dalian, China). 3-(4,5-Dimethylthiazol-2-yl)-2,5-diphenyltetrazolium bromide (MTT) was purchased from Sigma Aldrich (St. Louis, MO, USA). Melanin and GEM were also purchased from Sigma Aldrich (St. Louis, Mo, USA). 3,3'-Dioctadecyloxacarbocyanine perchlorate (DiO) was purchased from Heowns (Tianjin, China). Cell Counting Kit-8 (CCK-8) was purchased from Dojindo (Kyushu, Japan). All chemicals mentioned above were of analytical grade and used without further purification. All other solvents were of analytical or chromatographic grade.

\section{Preparation of GEM-Lip, Mel-Lip, and GEM-Mel-Lip}

The GEM-Mel-Lip was synthesized by the following procedure: lipid solution was prepared by dissolving a certain amount of lipid mixture with DPPC, DSPE-mPEG 2000, and cholesterol (55: $5: 40$ ) into $15 \mathrm{~mL}$ of trichloromethane. GEM was dissolved in DMSO solution, and was then mixed with the lipid solution. The mixture was volatized in a rotary evaporator under reduced pressure at $60{ }^{\circ} \mathrm{C}$. Melanin solution was prepared by dissolving melanin granule in $5 \mathrm{~mL}$ of ammonium hydroxide. Then, the obtained lipid film was hydrated with $5 \mathrm{~mL}$ of melanin solution to prepare the GEM-Mel-Lip by sonication at room temperature. GEM-Lip and Mel-Lip were prepared in a similar method. Subsequently, the formed GEM-Lip, Mel-Lip, and GEM-Mel-Lip were all extruded through $400 \mathrm{~nm}$ and $200 \mathrm{~nm}$ pore size polycarbonate membrane filters at room temperature. Finally, nanoliposomes with uniform particle size in nanoscale were achieved.

\section{Characterization of GEM-Mel-Lip}

Diameters of blank Lip, GEM-Lip, Mel-Lip, and GEM-Mel-Lip were measured by dynamic light scattering (DLS) at $25{ }^{\circ} \mathrm{C}$ using a Zetasizer Nano ZS (Malvern Instruments, UK). Polydispersity index (PDI) was calculated using software. The stability of GEM-Mel-Lip was also investigated by monitoring the size change of GEM-Mel-Lip for a two-week period. The UVvis spectra and zeta potential of GEM-Mel-Lip were investigated.
The morphology of GEM-Mel-Lip was observed by a transmission electron microscope (TEM, JEM-200CX, Jeol Ltd., Tokyo, Japan) after depositing the nanoliposomes on a carboncoated copper mesh grid. The content of GEM in the nanoliposomes was calculated by UV-vis spectrophotometer at $274 \mathrm{~nm}$. The encapsulation efficiency was calculated from the following formula: encapsulation efficiency (EE, \%) = (weight of drug loaded/total weight of drug put in GEM-Mel-Lip) $\times 100 \%$

\section{In vitro photothermal properties of the nanoliposomes}

The photothermal properties of blank Lip, Melanin, GEM-Lip, Mel-Lip and GEM-Mel-Lip were investigated by NIR irradiation at $808 \mathrm{~nm}\left(1.50 \mathrm{~W} \mathrm{~cm}^{-2}\right)$. The temperature changes were recorded every 30 seconds by a thermocouple in vitro.

\section{In vitro GEM release profiles}

The release profiles of GEM from GEM-Mel-Lip with or without NIR irradiation were evaluated by the dialysis method. Briefly, GEM-Mel-Lip were dispersed in PBS $(\mathrm{pH}=7.4)$ and transferred to dialysis bags (MWCO: $2000 \mathrm{Da}$ ). In the NIR group, GEM-MelLip was irradiated by an $808 \mathrm{~nm}$ laser $\left(1.50 \mathrm{~W} \mathrm{~cm}^{-2}\right)$ for $5 \mathrm{~min}$ before transferring to dialysis bags. Subsequently, the dialysis bags were immersed in centrifuge tubes containing $100 \mathrm{~mL}$ of PBS and incubated with continuous stirring for $30 \mathrm{~min}$ at $37^{\circ} \mathrm{C}$. The PBS outside the dialysis bags was replaced with fresh PBS. The ultraviolet absorption peak wavelength of GEM was $274 \mathrm{~nm}$. The concentration of the released GEM was measured, and then the release curve was obtained with time as the $x$-axis.

\section{In vitro cytotoxicity of melanin, GEM-Lip, Mel-Lip, and GEM- Mel-Lip}

The BxPC-3 cell line (human pancreatic cancer) was purchased from the Shanghai Branch of the Chinese Academy of Sciences (Shanghai, China) and cultured in RPMI-1640 substrate with $1.5 \mathrm{~g} \mathrm{~L}^{-1} \mathrm{NaHCO}_{3}, 2.5 \mathrm{~g} \mathrm{~L}^{-1}$ glucose and $0.11 \mathrm{~g} \mathrm{~L}^{-1}$ pyruvate in a humidified atmosphere of $5 \% \mathrm{CO}_{2}$ at $37{ }^{\circ} \mathrm{C}$. To study the cytotoxicity of Melanin, GEM-Lip, Mel-Lip, and GEM-Mel-Lip, BxPC-3 cells were firstly inoculated at a density of $5 \times 10^{4}$ cells into a 96-well plate and incubated for $48 \mathrm{~h}$. Subsequently, tested nanoliposomes with various concentrations were added immediately to the cells for another $24 \mathrm{~h}$. Cell viabilities were determined by CCK-8 assay. Five replicates were conducted for each group. The cytotoxicity of GEM-Lip, Mel-Lip, and GEMMel-Lip was also investigated under NIR irradiation $(808 \mathrm{~nm}$, $1.50 \mathrm{~W} \mathrm{~cm}^{-2}$ ).

\section{In vitro cellular uptake studies of GEM-Mel-Lip}

Profiles of cellular uptake of GEM-Mel-Lip were observed using confocal laser scanning microscopy (DMI 4000B, Leica Microsystems, Wetzlar, Germany). BxPC-3 cells were seeded on glassbottomed dishes for $24 \mathrm{~h}$ to form a confluent monolayer at $37{ }^{\circ} \mathrm{C}$. GEM-Mel-Lip was put in the dishes followed by different treatments and incubated for another $4 \mathrm{~h}$. Then, the glassbottomed dishes were viewed. In our study, DiO, a lipophilic fluorescent dye (Ex $484 \mathrm{~nm}$ and Em $501 \mathrm{~nm}$ ), was entrapped into 
lipid bilayers to indicate the localization of the nanoliposomes. The nuclei of the BxPC-3 cells were stained by blue DAPI (Ex $340 \mathrm{~nm}$ and $\mathrm{Em} 488 \mathrm{~nm}$ ).

\section{BxPC-3 tumor-bearing mice model}

All experiments were in accordance with the National Institute of Health Guide for the Care and Use of Laboratory Animals and conducted in line with protocols approved by the Tianjin First Center Hospital. To establish BxPC-3 tumor-bearing mice models, $2 \times 10^{6}$ BxPC- 3 cells were subcutaneously injected into the right lateral hind hip of BALB/C nude mice, which were purchased from Beijing Huafukang Biological Technology Co. Ltd. The size of the tumor was measured, and the volume of the tumor was calculated as $V=\mathrm{AB}^{2} / 2$, in which A represents the maximum diameter and $\mathrm{B}$ represents the minimum diameter of the tumor.

\section{In vivo antitumor efficacy of GEM-Mel-Lip}

To evaluate the antitumor efficacy of GEM-Mel-Lip, BxPC-3 tumor-bearing mice were treated with saline (control), GEMLip, Mel-Lip, and GEM-Mel-Lip $( \pm$ NIR) via tail vein injection when the tumor volume reached $\sim 100 \mathrm{~mm}^{3}$ (GEM or equivalent dosage: $50 \mathrm{mg} \mathrm{kg}^{-1}$ body weight). In NIR groups, the tumor area was irradiated with a laser $\left(808 \mathrm{~nm}, 1.50 \mathrm{~W} \mathrm{~cm}^{-2}, 1 \mathrm{~min} \times 3\right.$ times, 1 min interval). Each therapeutic group had four mice. Tumor size was measured by traceable digital vernier calipers every 3 days during a treatment cycle for 3 weeks. Subsequently, the mice were sacrificed, and the tumors were excised and weighed. Body weights of each group of mice were also monitored every 3 days. Finally, the tumor tissues in all of the above groups were stained by hematoxylin/eosin (H\&E) for histopathology analysis.

\section{Safety evaluation of GEM-Mel-Lip}

The mice were intravenously injected with saline and GEM-MelLip. The mice were killed after 1 day; the blood samples and major organs (heart, liver, spleen, lung, and kidney) were then collected. The serum biochemical index included AST, ALT, BUN, and CR was analyzed with the blood samples. Major organs were stained by H\&E for pathological observation.

\section{Statistical analysis}

Data were presented as the mean \pm standard deviation (SD), and the resulting values from each experiment were subjected to the paired two-tailed Student's $t$-test. The level of significance was defined at $p<0.01$.

\section{Conflicts of interest}

The authors declare no conflicts of interest.

\section{Acknowledgements}

This work was supported by the Chinese Foundation for Hepatitis Prevention and Control-TianQing Liver Disease
Research Foundation [grant number TQGB20170123] and the Tianjin Clinical Research Center for the Organ Transplantation Project [grant number 15ZXLCSY00070].

\section{References}

1 J. P. Neoptolemos, D. H. Palmer, P. Ghaneh, E. E. Psarelli, J. W. Valle, P. Hammel, T. Hackert, R. Jackson and M. W. Büchler, Comparison of adjuvant gemcitabine and capecitabine with gemcitabine monotherapy in patients with resected pancreatic cancer (ESPAC-4): a multicentre, open-label, randomised, phase 3 trial, Lancet, 2012, 389(10073), 1011-1024.

2 R. L. Siegel, K. D. Miller, S. A. Fedewa, D. J. Ahnen, R. G. S. Meester, A. Barzi and A. Jemal, Colorectal cancer statistics, 2017, Ca-Cancer J. Clin., 2017, 67(3), 177-193.

3 J. Kleeff, M. Korc, M. Apte, C. La Vecchia, C. D. Johnson, A. V. Biankin, R. E. Neale, M. Tempero, D. A. Tuveson, R. H. Hruban and J. P. Neoptolemos, Pancreatic Cancer, Nat. Rev. Dis. Primers, 2016, 2(5), 16022.

4 J. P. Neoptolemos, J. A. Dunn, D. D. Stocken, J. Almond, K. Link, H. Friess and M. W. Büchler, ESPAC-1: A European, randomised study to assess the roles of adjuvant chemotherapy and chemoradiation in resectable pancreatic cancer, Lancet, 2001, 358(9293), 1576-1585.

5 H. Oettle, P. Neuhaus, A. Hochhaus, J. T. Hartmann, K. Gellert, K. Ridwelski, M. Niedergethmann, C. Zülke, J. Fahlke, M. B. Arning, M. Sinn, A. Hinke and H. Riess, Adjuvant chemotherapy with gemcitabine and long-term outcomes among patients with resected pancreatic cancer: the CONKO-001 randomized trial, JAMA, 2013, 310(140), 1473-1481.

6 S. Hamsici, M. Sardan Ekiz, G. Cinar Ciftci, A. B. Tekinay and M. O. Guler, Gemcitabine Integrated Nano-Prodrug Carrier System, Bioconjugate Chem., 2017, 28(5), 1491-1498.

7 X. Zhao, K. Yang, R. Zhao, T. Ji, X. Wang, X. Yang, Y. Zhang, K. Cheng, S. Liu, J. Hao, H. Ren, K. W. Leong and G. Nie, Inducing enhanced immunogenic cell death with nanocarrier-based drug delivery systems for pancreatic cancer therapy, Biomaterials, 2016, 102, 187-197.

8 P. Zhang, W. Yi, J. Hou, S. Yoo, W. Jin and Q. Yang, A carbon nanotube-gemcitabine-lentinan three-component composite for chemo-photothermal synergistic therapy of cancer, Int. J. Nanomed., 2018, 13, 3069-3080.

9 A. Neesse, P. Michl, K. K. Frese, C. Feig, N. Cook, M. A. Jacobetz, M. P. Lolkema, M. Buchholz, K. P. Olive, T. M. Gress and D. A. Tuveson, Stromal biology and therapy in pancreatic cancer, Gut, 2011, 60(6), 861-868.

10 F. Sauvage, E. Fattal, W. Al-Shaer, S. Denis, E. Brotin, C. Denoyelle, C. Blanc-Fournier, B. Toussaint, S. Messaoudi, M. Alami, G. Barratt and J. VergnaudGauduchon, Antitumor activity of nanoliposomes encapsulating the novobiocin analog $6 \mathrm{BrCaQ}$ in a triplenegative breast cancer model in mice, Cancer Lett., 2018, 432, 103-111.

11 Y. Liu, F. Yang, C. Yuan, M. Li, T. Wang, B. Chen, J. Jin, P. Zhao, J. Tong, S. Luo and N. Gu, Magnetic 
Nanoliposomes as in situ Microbubble Bombers for Multimodality Image-Guided Cancer Theranostics, ACS Nano, 2017, 11(2), 1509-1519.

12 R. Sharma, N. Mody, V. Kushwah, S. Jain and S. P. Vyas, CType lectin receptor(s)-targeted nanoliposomes: an intelligent approach for effective cancer immunotherapy, Nanomedicine, 2017, 12(16), 1945-1959.

$13 \mathrm{Y}$. Matsumura and $\mathrm{H}$. Maeda, A new concept for macromolecular therapeutics in cancer chemotherapy: mechanism of tumoritropic accumulation of proteins and the antitumor agent smancs, Cancer Res., 1986, 46(12 Pt 1), 6387-6392.

14 N. Li, C. X. Zhang, X. X. Wang, L. Zhang, X. Ma, J. Zhou, R. J. Ju, X. Y. Li, W. Y. Zhao and W. L. Lu, Development of targeting lonidamine liposomes that circumvent drugresistant cancer by acting on mitochondrial signaling pathways, Biomaterials, 2013, 34(13), 3366-3380.

15 Y. Yu, Z. H. Wang, L. Zhang, H. J. Yao, Y. Zhang, R. J. Li, R. J. Ju, X. X. Wang, J. Zhou, N. Li and W. L. Lu, Mitochondrial targeting topotecan-loaded liposomes for treating drug-resistant breast cancer and inhibiting invasive metastases of melanoma, Biomaterials, 2012, 33(6), 1808-1820.

16 M. Yu, F. Guo, J. Wang, F. Tan and N. Li, PhotosensitizerLoaded pH-Responsive Hollow Gold Nanospheres for Single Light-Induced Photothermal/Photodynamic Therapy, ACS Appl. Mater. Interfaces, 2015, 7(32), 1759217597.

17 J. Lu, E. Choi, F. Tamanoi and J. I. Zink, Light-activated nanoimpeller controlled drug release in cancer cells, Small, 2010, 4(4), 421-426.

$18 \mathrm{~J}$. Davidsen, K. Jørgensen, T. L. Andresen and O. G. Mouritsen, Secreted phospholipase $\mathrm{A}(2)$ as a new enzymatic trigger mechanism for localised liposomal drug release and absorption in diseased tissue, Biochim. Biophys. Acta, 2003, 1609(1), 95-101.

19 M. Yu, X. Xu, Y. Cai, L. Zou and X. Shuai, Perfluorohexanecored nanodroplets for stimulations-responsive ultrasonography and $\mathrm{O}_{2}$-potentiated photodynamic therapy, Biomaterials, 2018, 175, 61-71.

20 V. Plassat, C. Wilhelm, V. Marsaud, C. Ménager, F. Gazeau, J. M. Renoir and S. Lesieur, Anti-Estrogen-Loaded Superparamagnetic Liposomes for Intracellular Magnetic Targeting and Treatment of Breast Cancer Tumors, Adv. Funct. Mater., 2011, 21(1), 83-92.

21 M. Yu, F. Guo, J. Wang, F. Tan and N. Li, Dual-targeting nanocarrier system based on thermosensitive liposomes and gold nanorods for cancer thermo-chemotherapy, $J$. Controlled Release, 2015, 215, 91-100.
22 M. Erkan, S. Hausmann, C. W. Michalski, A. A. Fingerle, M. Dobritz, J. Kleeff and H. Friess, The role of stroma in pancreatic cancer: diagnostic and therapeutic implications, Nat. Rev. Gastroenterol. Hepatol., 2012, 9(8), 454-467.

23 B. Farrow, D. Albo and D. H. Berger, The role of the tumor microenvironment in the progression of pancreatic cancer, J. Surg. Res., 2008, 149(2), 319-328.

24 L. Cheng, J. Liu, X. Gu, H. Gong, X. Shi, T. Liu, C. Wang, X. Wang, G. Liu, H. Xing, W. Bu, B. Sun and Z. Liu, PEGylated WS2 Nanosheets as a Multifunctional Theranostic Agent for in vivo Dual-Modal CT/Photoacoustic Imaging Guided Photothermal Therapy, Adv. Mater., 2014, 26, 1886-1893.

25 L. Wen, L. Chen, S. Zheng, J. Zeng, G. Duan, Y. Wang, G. Wang, Z. Chai, Z. Li and M. Gao, Ultrasmall Biocompatible $\mathrm{WO}_{3}-x$ Nanodots for Multi-Modality Imaging and Combined Therapy of Cancers, Adv. Mater., 2016, 28(25), 5072-5079.

26 H. Lin, X. Wang, L. Yu, Y. Chen and J. Shi, Two-Dimensional Ultrathin MXene Ceramic Nanosheets for Photothermal Conversion, Nano Lett., 2017, 17(1), 384-391.

27 Y. Liu, K. Ai, J. Liu, M. Deng, Y. He and L. Lu, DopamineMelanin Colloidal Nanospheres: An Efficient Near-Infrared Photothermal Therapeutic Agent for in vivo Cancer Therapy, Adv. Mater., 2013, 25(9), 1353-1359.

28 Q. Jiang, Z. Luo, Y. Men, P. Yang, H. Peng, R. Guo, Y. Tian, Z. Pang and W. Yang, Red blood cell membranecamouflaged melanin nanoparticles for enhanced photothermal therapy, Biomaterials, 2017, 143, 29-45.

29 R. H. Fang, Y. Jiang, J. C. Fang and L. Zhang, Cell membranederived nanomaterials for biomedical applications, Biomaterials, 2017, 128, 69-83.

30 J. G. Piao, L. Wang, F. Gao, Y. Z. You, Y. Xiong and L. Yang, Erythrocyte membrane is an alternative coating to polyethylene glycol for prolonging the circulation lifetime of gold nanocages for photothermal therapy, ACS Nano, 2014, 8(10), 10414-10425.

31 C. M. Hu, L. Zhang, S. Aryal, C. Cheung, R. H. Fang and L. Zhang, Erythrocyte membrane-camouflaged polymeric nanoparticles as a biomimetic delivery platform, Proc. Natl. Acad. Sci. U. S. A., 2011, 108(27), 10980-10985.

32 T. M. Allen, Ligand-targeted therapeutics in anticancer therapy, Nat. Rev. Cancer, 2002, 2(10), 750-763.

33 L. Zhang, D. Sheng, D. Wang, Y. Yao, K. Yang, Z. Wang, L. Deng and Y. Chen, Bioinspired Multifunctional Melanin-Based Nanoliposome for Photoacoustic/Magnetic Resonance Imaging-Guided Efficient Photothermal Ablation of Cancer, Theranostics, 2018, 8(6), 1591-1606. 